\title{
SLOPE OF THE ISGUR-WISE FUNCTION FROM A QSSR CONSTRAINT ON THE $\Upsilon B \bar{B}$ COUPLINGS
}

\author{
I.Caprini \\ Institute for Atomic Physics, POB MG 6 \\ Bucharest, Romania
}

\begin{abstract}
We derive optimal upper and lower bounds on the slope of the Isgur-Wise function at the zero recoil point in terms of the sum of the $\Upsilon B \bar{B}$ couplings, estimated recently from a $Q C D$ Spectral Sum Rule $(Q S S R)$. The problem is solved by means of a duality theorem in functional optimization. Optimal correlations between the slope and the convexity parameters of the Isgur-Wise function with the same input are also obtained.
\end{abstract}




\section{Introduction}

In the last time, there has been some interest in the investigation of the $b$-number form factor $F\left(q^{2}\right)$ of the $B$-meson

$$
<B\left(p^{\prime}\right)\left|V^{\mu}\right| B(p)>=\left(p+p^{\prime}\right)^{\mu} F\left(q^{2}\right)
$$

where $V^{\mu}=\bar{b} \gamma^{\mu} b$ and $q=p^{\prime}-p$. In the large quark mass limit, this form factor coincides with the renormalized Isgur-Wise function [1] $\xi(\omega), \omega=v \cdot v^{\prime}=1-q^{2} / 2 M_{B}^{2}$, describing the semileptonic decays of $B$ into $D$ and $D^{*}$ mesons. The values of the form factor in the physical region relevant for these decays $\left(\omega \geq 1\right.$, i.e. $q^{2}<$ 0 ) are of interest for the determination of the mixing matrix element $\left|V_{c b}\right|$ from experimental data. Various models for the Isgur-Wise function have been proposed in the last time in the frame of the heavy-quark effective theory (HQET). On the other hand, the possibility of obtaining model independent bounds on the values of $F\left(q^{2}\right)$, particularly on its slope at the origin (the charge radius) was outlined for the first time in [2]. These bounds follow from analyticity, unitarity and the asymptotic perturbative $Q C D$ expansion for the amplitude of the vacuum polarization due to the $V_{\mu}$ current. As noticed in the subsequent papers [3-6], the derivation [2] suffered from the drawback that it did not include the three upsilon poles present in the form factor $F$ below the $B \bar{B}$ threshold. The problem was correctly treated in $[7,8]$, where bounds on $F\left(q^{2}\right)$ were derived using as input only the position of the three upsilon poles below the $B \bar{B}$ threshold. Although the methods are different, the results obtained in [7] and [8] are both optimal. With a weak form of the unitarity [7], and using as input only the poles location, known from the experimental masses of the $\Upsilon$ resonances, the following bounds on the slope of the Isgur-Wise function were obtained

$$
-6 . \leq F^{\prime}(1) \leq 4.1
$$

where the derivative is with respect to $\omega$. The upper bound is actually not interesting, since it is much larger than the Bjorken upper bound -0.25 [2]. In [7] bounds on $F^{\prime}(1)$ were also calculated by assuming that the residua of $\Upsilon$ the poles are known. Using as input several particular values for the $\Upsilon B \bar{B}$ couplings, the conservative lower bound

$$
F^{\prime}(1) \geq-1.5
$$

was proposed in [7], but the authors stress upon the fact that the only rigorous lower bound on the slope of the $b$-number form factor at the origin is (1.2).

It is of interest to know whether this bound can be improved using only model independent information about the $\Upsilon B \bar{B}$ couplings. Recently, using a $Q C D$ Spectral Sum Rule $(Q S S R)$, Narison [9] obtained an estimate for the sum of the residua of the three upsilon resonances $\Upsilon(1 S), \Upsilon(2 S), \Upsilon(3 S)$ situated below the threshold $4 M_{B}^{2}$. However, in [9] this estimate was used only as an indication in choosing several

particular values for the $\Upsilon B \bar{B}$ couplings. Upper and lower bounds on $F^{\prime}(1)$ were then computed with the method presented in [7], for these specific values of the 
couplings. No attempt was made up to now to evaluate the real constraining power of the relation derived in [9], i.e. to calculate bounds on the slope of the Isgur-Wise function in terms of the sum of the upsilon pole residua.

In the present paper we address this specific problem. We derive upper and lower bounds on the slope $F^{\prime}(1)$ using as input the positions of the $\Upsilon$ poles and the sum of their residua. We also derive an optimal inequality relating the slope and the convexity parameters of the Isgur-Wise function, using the same input. These are the best model independent theoretical results which can obtained without choosing particular values for the $\Upsilon B \bar{B}$ couplings.

The paper is organized as follows: in the next Section we derive upper and lower bounds on the slope $F^{\prime}(1)$, using analyticity, unitarity, the $Q C D$ one-loop expansion for the polarization function and the sum of the $\Upsilon B \bar{B}$ couplings derived from $Q S S R$. We solve the problem by applying a duality theorem in functional optimization $[10,11]$. We also generalize the method to include higher derivatives of the form factor at the origin. The results, expressed as explicit inequalities involving the quantities of interest, are investigated numerically in Section 3.

\section{Derivation of the bound}

We consider the $b$-number form factor $F\left(q^{2}\right)$ defined in (1.1), which is a real-analytic function in the complex plane $t=q^{2}$, cut along the real axis from $4 M_{B}^{2}$ to infinity, except for $N=3$ poles below the $B \bar{B}$ threshold, corresponding to the states $\Upsilon(1 S), \Upsilon(2 S)$ and $\Upsilon(3 S)$. Their contribution to the form factor can be parametrized as $[7]$

$$
F(t)=F(0)+t \sum_{i=1}^{3} \frac{3 g_{\Upsilon_{i} B \bar{B}} f_{\Upsilon_{i}}}{M_{\Upsilon_{i}}^{2}-t-i \epsilon}+\ldots . .
$$

where $f_{\Upsilon_{i}}$ denote the coupling constants which govern the electronic widths of the $\Upsilon_{i}$-resonances

$$
\Gamma\left(\Upsilon_{i} \rightarrow(\gamma) \rightarrow e^{+} e^{-}\right)=f_{\Upsilon_{i}}^{2} M_{\Upsilon_{i}} \frac{4 \pi}{3} \alpha^{2}
$$

and $g_{\Upsilon_{i} B \bar{B}}$ are the coupling constants of the $\Upsilon_{i}$ resonances to the $B \bar{B}$ system. As in [7] we denote by $\eta_{i}$ the product of the above coupling constants

$$
\eta_{i}=3 g_{\Upsilon_{i} B \bar{B}} f_{\Upsilon_{i}}, \quad i=1,2,3 .
$$

By using a $Q S S R$ for the vertex function, Narison [9] estimated recently the sum of these couplings:

$$
\sum_{i=1}^{3} \eta_{i}=\alpha=-0.224
$$

We shall take this model independent relation as input in deriving bounds on the derivative of the Isgur-Wise function at the origin. We use also the normalization condition

$$
F(0)=1
$$


and the integral inequality [7]

$$
\frac{n_{f}}{12} \frac{1}{16 M_{B}^{2} \chi\left(Q^{2}\right)} \int_{1}^{\infty} \frac{(y-1)^{3 / 2}}{y^{3 / 2}\left(y+Q^{2} / 4 M_{B}^{2}\right)^{2}}\left|F\left(4 M_{B}^{2} y\right)\right|^{2} d y \leq 1
$$

where $y=\frac{t}{4 M_{B}^{2}}$. Here

$$
\chi\left(Q^{2}\right)=\frac{N_{c}}{2 \pi^{2}} \int_{0}^{1} \frac{x^{2}(1-x)^{2} d x}{m_{b}^{2}+Q^{2} x(1-x)}
$$

is the $Q C D$ one-loop level expression of the derivative of the polarization function, for spacelike values $Q^{2} \geq 0$, and $N_{c}$ is the number of colours. In deriving (2.6) it was assumed [7] that the unitarity sum for the polarization function is saturated with the lowest $B \bar{B}$ states and that the contribution of the intermediate states $B^{+} B^{-}, B^{0} \bar{B}^{0}$ and $B_{s}^{0} \bar{B}_{s}^{0}$ is the same in the limit where the light quark mass differences are small. The stronger version of unitarity used in $[2,6,8]$, which requires additional dynamical assumptions, beyond $H Q E T$, will not be adopted below.

In the inequality (2.6) the spacelike momentum $Q^{2}$ enters as a parameter. The dependence of the results on the choice of $Q^{2}$, as well as on the $Q C D$ sum rule (dispersion relation) written for the polarization function was discussed in [8]. In what follows we shall take the particular value $Q^{2}=0$. Then (2.6) becomes

$$
\frac{5 n_{f}}{16 N_{c}} \int_{1}^{\infty}(y-1)^{3 / 2} y^{-7 / 2}|F(y)|^{2} d y \leq 1
$$

We write this inequality in a canonical form by performing the conformal mapping

$$
z=\frac{\sqrt{1-y}-1}{\sqrt{1-y}+1}
$$

which applies the cut y-plane onto the interior of the unit disk $|z|<1$, such that $z(0)=0$ and the beginning of the cut, $y=1$ is transformed into $z=-1$. In the new variable the positions of the $\Upsilon$ poles are denoted by $z_{i}$ and the residua of the form factor $F(z)$ at these poles can be written as

$$
r_{i}=\left.\left(z-z_{i}\right) F(z)\right|_{z=z_{i}}=\eta_{i} z_{i} \frac{1-z_{i}}{1+z_{i}}
$$

with $\eta_{i}$ defined in (2.3). Also, the inequality (2.8) becomes

$$
\frac{1}{2 \pi} \int_{0}^{2 \pi} w(\theta)|F(\theta)|^{2} d \theta \leq 1
$$

where

$$
w(\theta)=\frac{5 \pi n_{f}}{48} \cos ^{4} \frac{\theta}{2} \sin \frac{\theta}{2}
$$


is a nonnegative weight. Of interest for us are the slope and the convexity parameters of the form factor

$$
\rho^{2}=-F^{\prime}(1), c=\frac{1}{2} F^{\prime \prime}(1) .
$$

the derivatives being with respect to $\omega$, which are related to the derivatives of $F$ with respect to $z$ through

$$
F_{z}^{\prime}(0)=-8 \rho^{2}, F_{z}^{\prime \prime}(0)=128 c-32 \rho^{2}
$$

We shall obtain upper and lower bounds on the derivatives of $F$, using as input the analyticity of the form factor in the cut $t$-plane, except for the $\Upsilon$ poles, and the conditions (2.4), (2.5) and (2.6) ( the last one being written alternatively as (2.11)).

In order to bring the problem to a standard form, we consider first the function $[2,7]$

$$
\phi(z)=\phi(0)(1+z)^{2} \sqrt{1-z}, \quad \phi(0)=\frac{1}{16} \sqrt{\frac{5 \pi n_{f}}{2 N_{c}}},
$$

which is analytic and nonzero inside the unit disk $|z|<1$ and whose modulus on the boundary is related to the weight $w(\theta)$ given in (2.12) through

$$
|\phi(\theta)|=\sqrt{w(\theta)}
$$

Let us define now the new function

$$
g(z)=F(z) \phi(z) B(z)
$$

where $F$ is the form factor and

$$
B(z)=\prod_{i=1}^{3} \frac{z-z_{i}}{1-z_{i}^{*} z} .
$$

is a product of so-called Blaschke factors [10] (in our case, to a good approximation, $z_{i}^{*}=z_{i}$ ). It is clear that the function $g$ is analytic in $|z|<1$, the poles of $F$ being canceled by the corresponding zeros of $B$. Moreover, the constraints (2.4), (2.5) and (2.11) on the form factor can be written immediately in terms of the function $g$. Thus, using (2.10) and (2.15) we have

$$
g\left(z_{i}\right)=r_{i} \phi\left(z_{i}\right)\left[\frac{B(z)}{z-z_{i}}\right]_{z=z_{i}}
$$

and the condition (2.4) becomes

$$
\sum_{i=1}^{3} \gamma_{i} g\left(z_{i}\right)=\alpha
$$


Here the weights $\gamma_{i}$ are defined as

$$
\gamma_{i}=-\frac{1}{\phi(0) z_{i}\left(1+z_{i}\right)\left(1-z_{i}\right)^{3 / 2} \hat{B}_{i}},
$$

with

$$
\hat{B}_{i}=\left[\frac{B(z)}{z-z_{i}}\right]_{z=z_{i}} .
$$

As concerns the conditions (2.5) and (2.11), they become

$$
g(0)=\phi(0) B(0)
$$

and, respectively

$$
\|g\|_{L^{2}} \equiv\left\{\frac{1}{2 \pi} \int_{0}^{2 \pi}|g(\theta)|^{2} d \theta\right\}^{1 / 2} \leq 1
$$

where we took into account (2.16) and the fact that the Blaschke factors have modulus equal to 1 along the unit circle. Using a standard terminology [10], (2.24) means that the function $g$ belongs to the unit sphere of the Hilbert space $H^{2}$.

We express also the derivatives of $g(z)$ at the origin in terms of the derivatives of $F(z)$, which are further related to the slope and convexity parameters through (2.14). Using the relations

$$
\phi^{\prime}(0)=\frac{3}{2} \phi(0), \quad \phi^{\prime \prime}(0)=-\frac{\phi(0)}{4},
$$

which follow from (2.15), we obtain

$$
\begin{gathered}
g^{\prime}(0)=\phi(0) B(0)\left[F^{\prime}(0)+\frac{3}{2}+\frac{B^{\prime}(0)}{B(0)}\right] \\
g^{\prime \prime}(0)=\phi(0) B(0)\left\{F^{\prime \prime}(0)+F^{\prime}(0)\left[3+2 \frac{B^{\prime}(0)}{B(0)}\right]-\frac{1}{4}+3 \frac{B^{\prime}(0)}{B(0)}+\frac{B^{\prime \prime}(0)}{B(0)}\right\},
\end{gathered}
$$

where we took into account (2.5) and the derivatives of $B$ follow from (2.18).

We pass now to the calculation of bounds on the derivatives of the analytic function $g$ in the origin, using as input the constraints (2.20), (2.23) and (2.24). We first consider only the slope of the form factor, i.e. the derivative $g^{\prime}(0)$. As will be clear, the generalization to higher derivatives is straightforward.

In order to solve the problem we shall apply a method based on a norm minimization over a convex set in $H^{2}$ and a duality theorem in functional optimization $[10,11]$. A similar technique was applied in [12] for other problems in particle physics. More precisely, let us consider the following set of functions:

$$
K=\left\{g \in H^{2} \mid g(0), g^{\prime}(0) \text { given, } \quad \sum_{i=1}^{3} \gamma_{i} g\left(z_{i}\right)=\alpha .\right\}
$$


It is clear that $K$ is a convex set in the Hilbert space $H^{2}$. In defining it, we took into account the constraint (2.20) and the fact that $g(0)$ is known, according to (2.23). In addition, we ascribed to the derivative $g^{\prime}(0)$, which is the quantity of interest, a definite value, for the moment arbitrary.

We have still to impose the condition (2.24) and this will restrict the allowed range of the unknown parameter $g^{\prime}((0)$. More precisely, if this parameter is correctly chosen, all the functions in the set $K$ will have $L^{2}$ norms less than or equal to 1 . If, on the other hand, $g^{\prime}(0)$ is outside the allowed range, the $L^{2}$ norms of all the functions in $K$ will be greater than 1 , and this will be true also for the smallest of these norms. It follows that the inequality

$$
\min _{g \in K}\|g\|_{L^{2}} \leq 1
$$

where the l.h.s. denotes the minimal $L^{2}$ norm over the set $K$, describes the optimal range of the parameter $g^{\prime}(0)$. As we shall see, the minimum norm (2.28) will depend explicitely on the specific information used in the definition of $K$, which consists from the values $g(0), g^{\prime}(0)$ and the real number $\alpha$. This will allow us to obtain explicitely rigorous upper and lower bounds on the unknown value $g^{\prime}(0)$ in terms of the value $g(0)$ and of the parameter $\alpha$.

Having reduced the problem to the calculation of a minimum norm, we resort for solving it to a duality theorem in functional analysis $[10,11]$. Usually, a duality theorem relates a minimization over an abstract space to a maximization in the dual space, which is often simpler than the original one. More precisely, if $K$ is a convex set in the Hilbert space $H^{2}$ and $g$ denotes an arbitrary function belonging to $K$, the theorem of interest for us has the form $[10,11]$ :

$$
\min _{g \in K}\|g\|_{L^{2}}=\sup _{G \in L^{2},\|G\|_{L^{2}} \leq 1}\left|-\sup _{g \in K} \frac{1}{2 \pi i} \oint_{|z|=1} G(z) g(z) d z\right| \text {. }
$$

Here the supremum in the right hand side is calculated upon all the complex functions $G$ defined on the boundary of the unit circle, of $L^{2}$ norms less than 1 . In writing (2.29) we took into account the fact that the dual of the space $L^{2}$ is again $L^{2}$.

We have first to calculate the supremum over $g \in K$ in the r.h.s. of this equation. In a standard terminology [10] this supremum is called the support functional of the convex $K$. Of course, of interest are only the finite values of the support functional. This clearly restricts the form of the the functions $G(z)$ taken into account in the functional optimization. As we mentioned above, the function $G$ entering the maximization (2.29) is a complex function of $L^{2}$ norm less than 1, being in general the boundary value of a function having an analytic part inside the unit disk, and possible interior singularities. By residua theorem, these singularities pick up in the integral (2.29) the values of $g$ at some interior points. It is clear that these values must be known, according to the definition of the set $K$, otherwise the supremum 
upon $g \in K$ would be uncontrolably large. By applying this argument to the particular set defined above we see that the functions $G$ yielding a finite support functional of $K$ have the general form

$$
G(z)=\frac{a_{1}}{z}+\frac{a_{2}}{z^{2}}+\sum_{i=1}^{3} \frac{b_{i}}{z-z_{i}}+Q(z)
$$

where the residua $a_{1}, a_{2}, b_{i}$ and the analytic function $Q(z)$ are arbitrary. By inserting this expression in (2.29) we obtain

$$
\min _{g \in K}\|g\|_{L^{2}}=\sup _{G \in H^{2},\|G\|_{L^{2}} \leq 1} \sup _{g \in K}\left|a_{1} g(0)+a_{2} g^{\prime}(0)+\sum_{i=1}^{3} b_{i} g\left(z_{i}\right)\right|,
$$

where we applied the residua theorem, which implies in particular that the analytic function $Q$ does not contribute.

It is clear that the supremum with respect to $g$ in the r.h.s. of this equation is finite only if the residua $b_{i}$ are proportional to the weigths $\gamma_{i}$ entering the constraint in the definition of $K$. We must have therefore

$$
b_{i}=a_{3} \gamma_{i}
$$

where $a_{3}$ is an arbitrary parameter, along with $a_{1}$ and $a_{2}$. Then (2.31) becomes

$$
\min _{g \in K}\|g\|_{L^{2}}=\sup _{\left\{a_{i}\right\}}\left[a_{1} g(0)+a_{2} g^{\prime}(0)+a_{3} \alpha\right]
$$

where the parameters $a_{i}$ entering the maximization in the r.h.s. must satisfy the condition that the $L^{2}$ norm of $G$ is less than 1. By performing the Fourier analysis of the function $G$ of the form (2.30) we obtain after a straightforward calculation the quadratic constraint

$$
\left(a_{1}+a_{3} \sum_{i=1}^{3} \gamma_{i}\right)^{2}+\left(a_{2}+a_{3} \sum_{i=1}^{3} \gamma_{i} z_{i}\right)^{2}++a_{3}^{2} \sum_{i, k=1}^{3} \frac{\gamma_{i} \gamma_{k} z_{i}^{2} z_{k}^{2}}{1-z_{i} z_{k}} \leq 1
$$

which must be satisfied by the parameters $a_{i}$.

The linear optimization problem (2.33) with the quadratic constraint (2.34) can be solved immediately, by means of a linear change of variable which brings this constraint to a diagonal form. The solution can be written in a compact form, and using (2.28) we finally obtain the inequality

$$
\left\{g^{\prime 2}(0)+g^{2}(0)+\frac{1}{\lambda}\left[\alpha-g(0) \sum_{i=1}^{3} \gamma_{i}-g^{\prime}(0) \sum_{i=1}^{3} \gamma_{i} z_{i}\right]^{2}\right\}^{\frac{1}{2}} \leq 1
$$

where

$$
\lambda=\sum_{i, k=1}^{3} \frac{\gamma_{i} \gamma_{k} z_{i}^{2} z_{k}^{2}}{1-z_{i} z_{k}}
$$


Using (2.23) and (2.26) the inequality (2.35) can be written in terms of $F(0)$ and $F^{\prime}(0)$, allowing one to calculate upper and lower bounds on the slope of the IsgurWise function using as input the sum , $\alpha$, of the $\Upsilon$ pole residua. The numerical results are presented in Section 3.

Before closing this section we mention that the technique applied above can be generalized in a straightforward way as to include higher derivatives of the form factor in the origin. This can be done by a suitable definition of the convex set $K$ and a proper choice of the function $G$ used in the duality theorem (2.29). Instead of (2.27) we now define

$$
K=\left\{g \in H^{2} \mid g(0), g^{\prime}(0), g^{\prime \prime}(0) \text { given, } \quad \sum_{i=1}^{3} \gamma_{i} g\left(z_{i}\right)=\alpha .\right.
$$

and the function $G$ of (2.30) is replaced by

$$
G(z)=\frac{a_{1}}{z}+\frac{a_{2}}{z^{2}}+\frac{a_{3}}{z^{3}}+\sum_{i=1}^{3} \frac{b_{i}}{z-z_{i}}+Q(z) .
$$

A calculation similar to the one presented above leads to the following inequality involving the first two derivatives of the form factor:

$$
\left\{\frac{g^{\prime \prime 2}(0)}{4}+g^{\prime 2}(0)+g^{2}(0)+\frac{1}{\xi}\left[\alpha-g(0) \sum_{i=1}^{3} \gamma_{i}-g^{\prime}(0) \sum_{i=1}^{3} \gamma_{i} z_{i}-\frac{g^{\prime \prime}(0)}{2} \sum_{i=1}^{3} \gamma_{i} z_{i}^{2}\right]^{2}\right\}^{\frac{1}{2}} \leq 1
$$

where

$$
\xi=\sum_{i, k=1}^{3} \frac{\gamma_{i} \gamma_{k} z_{i}^{3} z_{k}^{3}}{1-z_{i} z_{k}}
$$

The inequality (2.39), written in terms of the slope and convexity of the Isgur-Wise function by means of $(2.23),(2.26)$ and $(2.14)$, yields a rigorous correlation between these parameters and the sum $\alpha$, of the $\Upsilon$ poles residua. We shall apply it in the next Section for testing specific models of the HQET.

\section{$3 \quad$ Numerical results and conclusions}

In our analysis we took $M_{B}=5.3 \mathrm{GeV}$ and varied $m_{b}$ in the range $4.7-5.3 \mathrm{GeV}$. We first calculate upper and lower bounds on the derivative $F^{\prime}(1)$, using (2.35). The results are presented in Table 1, for several values of the parameter $\alpha$ in the constraint (2.4). 


\begin{tabular}{|c|c|c|}
\hline$\alpha$ & $F^{\prime}(1)_{\min }$ & $F^{\prime}(1)_{\max }$ \\
\hline-2.240 & -3.05 & 4.30 \\
\hline-0.272 & -3.91 & 3.77 \\
\hline-0.224 & -4.13 & 3.61 \\
\hline-0.0224 & -4.23 & 3.55 \\
\hline 0. & -4.23 & 3.53 \\
\hline 0.224 & -4.36 & 3.45 \\
\hline 0.672 & -4.55 & 3.29 \\
\hline 2.240 & -5.15 & 2.59 \\
\hline
\end{tabular}

Table 1: Upper and lower bounds on the slope of the Isgur-Wise function for various values of the sum (2.4) of the $\Upsilon$ pole residua.

In Table 1 we investigated for completeness several values of $\alpha$, up to ten times larger than the value proposed in [9] and given in (2.4). Values with opposite sign were considered as well. Taking $\alpha=-0.224$ we obtain

$$
-4.13 \leq F^{\prime}(1) \leq 3.61
$$

which slightly improve the values (1.2) and is the best bound on the slope of the $b$-number form factor, obtained using only model independent information about the residues.

We consider now the inequality (2.39) relating the slope and the convexity parameters of the Isgur-Wise function to the sum of the $\Upsilon B \bar{B}$ couplings. According to (2.39), the domain of the allowed values for these parameters is the interior of an ellipsa in the plane $\rho^{2}-c$. It is of interest to establish the compatibility of some particular models of $H Q E T$ with this rigorous inequality. For illustration, we consider the following parametrizations proposed in [9]:

$$
\begin{gathered}
F(\omega) \simeq 1+F^{\prime}(1)(\omega-1), \\
F(\omega) \simeq \exp \left\{F^{\prime}(1)(\omega-1)\right\}, \\
F(\omega) \simeq\left\{\frac{1}{2}(1+\omega)\right\}^{2 F^{\prime}(1)}, \\
F(\omega) \simeq \frac{2}{1+\omega} \exp \left\{\left[2 F^{\prime}(1)+1\right] \frac{\omega-1}{\omega+1}\right\} .
\end{gathered}
$$

These parametrizations, with the choice $F^{\prime}(1) \simeq-1.0$, were used in [9] for a new determination of the mixing angle $V_{c b}$. They correspond, respectively, to the following expressions for the convexity parameters:

$$
\begin{gathered}
c=0 . \\
c=\frac{1}{2}\left[F^{\prime}(1)\right]^{2}
\end{gathered}
$$




$$
\begin{gathered}
c=\frac{1}{4} F^{\prime}(1)\left[2 F^{\prime}(1)-1\right] \\
c=\frac{1}{2}\left[F^{\prime}(1)^{2}-F^{\prime}(1)-\frac{1}{4}\right] .
\end{gathered}
$$

In order to test the compatibility of the models (3.2) with the constraint (2.4), we calculate the derivatives $g^{\prime}(0)$ and $g^{\prime \prime}(0)$ using the relations (2.14) and (2.26) and we insert them in (2.39). The result of this calculation is given in Table 2, which indicates the l.h.s. of (2.39) denoted by $L H S$, for several $\mathrm{v}$ alues of $\rho^{2}$ and the parameter $c$ computed according to (3.3) for each specific model (3.2). The two columns correspond to $\alpha=-0.224$ as proposed in [9], and also to a value three times larger, respectively. Unlike the bounds on the slope given in Table 1, which had a slow variation with $\alpha$, the results are now quite sensitive to the sum of the pole residua. According to (2.39), a result less than 1 is expected to occur, if the parametrizations (3.2) are consistent with the constraint (2.4). However, as seen from Table 2 , for $\rho^{2}=1$ a value greater than 1 is obtained for all the models (3.2), which shows that these models are not compatible with the relation (2.4) proved in [9]. This throws some doubt on the determination of $V_{c b}$ made in [9], which was based on the models (3.2) with the choice $\rho^{2}=1$. On the other hand, for $\rho^{2}=0.5$, the models (3.2) turn out to be consistent with the relation (2.4), while they fail to be so if the value of $\alpha$ is increased by a factor of 3 .

\begin{tabular}{|c|c|c|c|}
\hline$\rho^{2}$ & $c$ & $\begin{array}{c}\text { LHS } \\
\alpha=-0.224\end{array}$ & $\begin{array}{c}\text { LHS } \\
\alpha=-0.672\end{array}$ \\
\hline 1. & 0. & 1.65 & 1.95 \\
\hline 1. & 0.5 & 1.37 & 1.78 \\
\hline 1. & 0.75 & 1.39 & 1.83 \\
\hline 1. & 0.875 & 1.44 & 1.89 \\
\hline 0.5 & 0. & 0.91 & 1.29 \\
\hline 0.5 & 0.125 & 0.87 & 1.28 \\
\hline 0.5 & 0.25 & 0.86 & 1.30 \\
\hline 0.5 & 0.25 & 0.86 & 1.30 \\
\hline
\end{tabular}

Table 2 : Left hand side of (2.39), denoted as $L H C$, for $\rho^{2}=1$ and $\rho^{2}=0.5$, and $c$ computed from (3.3) for the models (3.2). The two columns correspond to $\alpha=-0.224$ and to a value three times larger.

In conclusion, in the present paper we succeeded to fully exploit the constraining power of the $Q S S R$ relation (2.4) between the $\Upsilon B \bar{B}$ couplings, derived in [9]. The problem was solved by means of some powerful techniques in functional optimization.

The results show that the relation (2.4) has a rather weak constraining power on the slope of the Isgur-Wise function. In particular, it slightly improved the lower bound on $F^{\prime}(1)$ from the value -6 , when no information on the $\Upsilon B \bar{B}$ couplings 
is used, to the value -4.13 , which is still very generous. On the other hand, the constraint (2.4) is able to produce nontrivial correlations between the slope and the convexity parameters of the form factor. The relation (2.39) derived in the present paper turns out to be an useful tool for investigating the compatibility of the specific models of the quark theory with analyticity, unitarity and $Q C D$.

\section{References}

[1] N.Isgur and M.B.Wise, Phys.Lett.B232(1989) 113; B237(1990) 523

[2] E.de Rafael and J.Taron, Phys.Lett.B282(1992) 215

[3] 3 A.F.Falk, M.Luke and M.B.Wise, Phys.Lett.B299(1993) 123

[4] B.Grinstein and P.F.Mende, Phys.Lett.B299(1993) 127

[5] 5 C.E.Carlson et al Phys.Lett. B299 (1993) 133.

[6] C.A.Dominguez, J.Korner and D.Pirjol, Phys.Lett. B301 (1993) 257.

[7] E.de Rafael and J.Taron, preprint OHSTPY-HEP-T-93-003, CPT-93/P.2908 (1993).

[8] I.Caprini, Z.Phys.C 61 (1994) 651.

[9] S.Narison, Phys.Lett. B325 (1994) 197.

[10] P.Duren, Theory of $H^{p}$ Spaces, New York: Academic Press, 1970.

[11] D.G.Luenberger, Optimization by Vector Space Methods, New York: Wiley, 1968.

[12] I.Caprini and P.Dita, J.Phys.A:Math.Gen.14 (1980) 1271; I.Caprini, I.Guiasu and E.E.Radescu, Phys.Rev. D25 (1982) 1808. 